\title{
EDUCACIÓN
}

\section{Tutoría académica en la Facultad de Odontología de la Universidad Nacional de Asunción}

\author{
Stela Mary Benítez de Focardell ${ }^{1}$
}

\section{Resumen}

Introducción: La tutoría académica es un factor de calidad y una exigencia del Espacio Europeo de Educación Superior. Forma parte del sistema de gestión de calidad de la educación. Es el proceso de acompañamiento personal y académico cuyo objetivo es mejorar el rendimiento académico, solucionar problemas escolares, desarrollar hábitos de estudio, trabajo, reflexión y convivencia social. Nació como alternativa para reducir el índice de deserción y reprobación, en los centros educativos. Y especialmente para enfocar la educación hacia la formación integral del estudiante; para lo cual se debe realizar un trabajo comprometido y evaluar con juicio crítico todo el proceso de formación del discente. La mayor cobertura y acceso a la educación superior trajo consigo un gran desafío que es la formación integral del discente para una sociedad exigente, cada vez más competitiva y tecnológica. Considerando esto, en fecha 30 de mayo del año 2011, por Resolución № 239 se creó en la Facultad de Odontología de la Universidad Nacional de Asunción el Programa Institucional de Tutoría Académica como un plan estratégico para la formación integral del estudiante. Su objetivo fue elevar la calidad del proceso educativo, a través de la atención personalizada de los discentes, con miras a identificar tempranamente problemas que influyen en su desempeño social y rendimiento académico. Al mejorar las condiciones de aprendizaje y favoreciendo el desarrollo de hábitos, valores y actitudes que contribuyan a la formación integral de su persona, se promueve su crecimiento profesional, social y humano. Al ser considerada, la tutoría, como factor de calidad de la educación, debe cumplir con una serie de requisitos para desempeñar cabalmente su función; para lo cual es importante conocer la opinión que tienen del programa, los actores principales, que son los estudiantes; para lo cual, en el año 2014, se realizó una investigación, descriptiva de corte trasversal, con enfoque cuantitativo.

Objetivo: Determinar la percepción de los estudiantes de odontología, sobre la implementación del programa de tutoría académica.

Material y Método: La participación fue voluntaria. Formaron parte del estudio, 73 estudiantes, de primer al tercer curso, quienes fueron en ese entonces, los que habían pasado por el programa. Se aplicó un cuestionario

1. Facultad de Odontología, Universidad Nacional de Asunción, Paraguay.

E-mail: stelamary@hotmail.es

DOI: $10.26885 /$ rcei.foro.2017.96 


\section{Tutoría académica en la Facultad de Odontología de la UNA. Benítez de Focardell}

cerrado, autoadministrado. En la encuesta aplicada, se midió la empatía del tutor, la capacidad tutorial, el nivel de satisfacción de los alumnos en relación al programa de tutoría, la capacidad de orientación del tutor en relación al conocimiento de la institución, la disposición de los tutores hacia los alumnos, el tiempo brindado al tutorado, los temas tratados durante los encuentros y conocimiento del tutor en cuanto a manejo grupal e individual. La capacidad de orientación del tutor en relación al conocimiento de la institución, se refiere a si el tutor pudo aclarar las dudas de su tutorado en relación a las normativas de la institución y si su orientación fue acorde a dichas disposiciones, o si en algún momento se presentó alguna dificultad que escapó de su campo de acción, este lo supo llevar a la instancia correspondiente. Para la medición se consideró, respuesta: menor a 50\%: insuficiente; de 50 a 74 \%: bueno, de 75 a 90\%: muy bueno; mayor de 90\%: excelente. Los resultados fueron asentados en una planilla de Excel para luego ser analizado en el programa de Epi-Info, versión 3.5.4.

Resultados: El nivel de empatía del tutor fue bueno (59\%). La dificultad de relacionamiento 20,5\%; capacidad en cuanto a conocimientos de técnicas sobre asesoría individual o grupal, 63\%. La percepción de los estudiantes, en cuanto a la capacidad del tutor sobre el conocimiento de normativas institucionales es buena, (67,5\%). Disposición en cuanto a tiempo brindado fue buena, $(62,8 \%)$. Trato cordial, 78\%. El 54,8\% afirmó que el tutor no mostró interés en conocer las dificultades que tuvo el alumno y que en el $80 \%$ de los casos no hubo comunicación permanente. Los temas más tratados, fueron los académicos. Si bien, los tutorados consideran que el conocimiento del tutor sobre las normativas de la institución es bueno, este debería ser excelente, puesto que el tutor debe estar capacitado para orientar sobre todo en lo relacionado a las normativas institucionales. También es preocupante, que $79,5 \%$ de los tutorados haya manifestado que no pudo comunicarse con el tutor en forma permanente; porque este debe estar en comunicación constante o por lo menos localizable para el tutorado, de manera que si este tiene algún problema pueda concertar un encuentro y auxiliarlo en sus dudas. La calificación de la capacidad tutorial, fue deficiente $(<50 \%)$, esto es alarmante, puesto que la función del tutor es brindar al alumno atención personalizada y disipar dudas relacionadas al ámbito educativo y por qué no, familiares y sociales. La empatía, si bien es buena, (59\%), debería mejorar para lograr los objetivos del programa, porque si la relación no es la adecuada entre los protagonistas, es difícil que el alumno tenga la confianza suficiente para exponer sus dudas o problemas. Lo ideal sería lograr un 100\% de empatía con el alumno, para que este pueda hablar en un clima de confianza y cordialidad. Como el programa de Tutoría Académica es nuevo, existen muchos tópicos que se deben mejorar y reprogramar, así lo establecen los resultados obtenidos. Apremia trabajar en las mejoras, sobre todo, considerando los beneficios que la tutoría puede ejercer en la formación integral de los estudiantes, desde el punto de vista 
profesional y personal; disminuyendo los índices de reprobación, rezago y deserción, aumentando así eficiencia terminal en la formación del discente. Con este programa se logró minimizar el acúmulo de alumnos en los cursos superiores, al disminuir las repitencias. Esto favoreció las prácticas clínicas del alumno porque disminuyendo el número de discentes se pudo aumentar las horas de prácticas clínicas, porque el alumno puede ocupar el sillón de prácticas en forma individual y no en dupla; como lo hace en caso de que el número de alumnos rebase el número de sillones.

Palabras clave: tutoría académica, odontología, Universidad Nacional de Asunción.

\section{Referencias}

ANUIES. (2000). Programas Institucionales de Tutoría. Una propuesta de la AUNIES para su organización y funcionamiento en las instituciones de educación superior (2a ed. correg.). Recuperado de http://evirtual.uaslp. $\mathrm{mx} / \mathrm{FCQ} /$ tutorias/Documentos\%20compartidos/INTRODUCCION/ PROGRAMAS\%20INSTITUCIONALES\%20DE\%20tutoria_anuies. pdf

ANUIES. (2002). ANUIES: sistema tutorial. Recuperado de: http://www. tutoria.unam.mx/sites/default/files/11-anuies1abril.pdf.

ANUIES Tutorías

Castaño, E. P., Castañeda, E. A. \& Blanco, A. F. (2012). Competencias para la tutoría: experiencia de formación con profesores universitarios. Revista de Docencia Universitaria, 10(2), 193-210. Recuperado de: https:// polipapers.upv.es/index.php/REDU/article/view/6103

Gómez-Collado, E.M. (2012). La percepción de los estudiantes sobre el Programa de Tutoría Académica. Recuperado de http://www.scielo.org.mx/pdf/ conver/v19n58/v19n58a9.pdf

López, M. I., González, V. P. \& Velasco, Q. J. (2013). Ser y ejercer de tutor en la universidad. La misión actual de la universidad, 11(2), 107-134. 